\title{
Loss of fractured part of a proximally migrated esophageal stent through vomiting
}

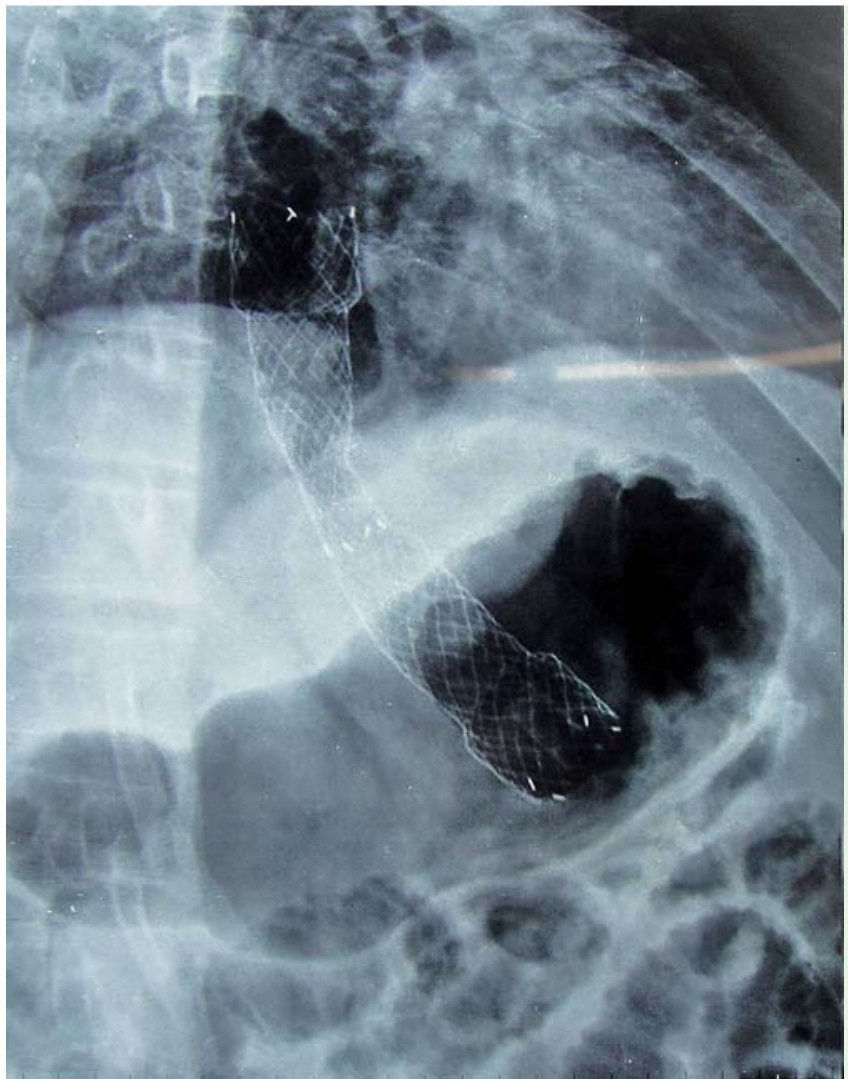

Fig. 1 Anteroposterior (AP) chest radiograph view at initial stent placement in a 47-yearold man with advanced gastroesophageal adenocarcinoma.
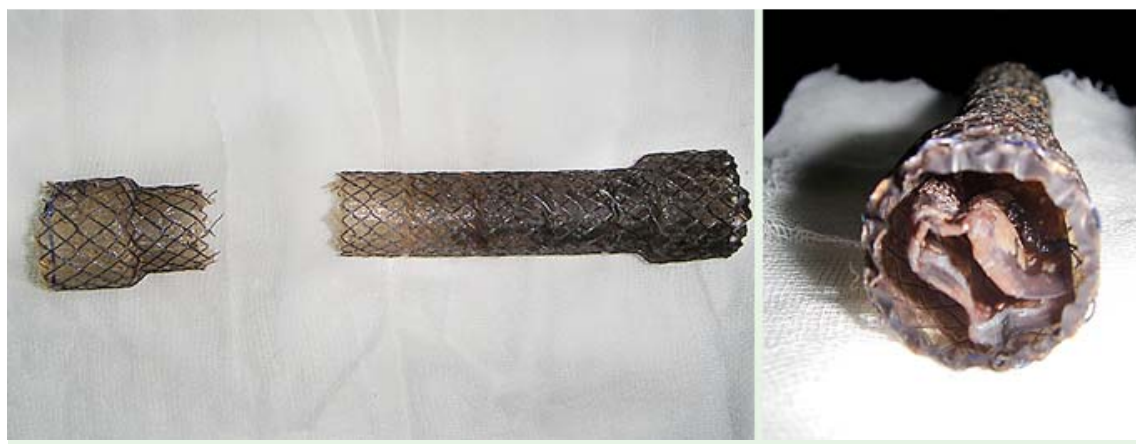

Fig. 3 Two fractured stent segments: the smaller proximal part was vomited out by patient and the longer distal part was retrieved endoscopically.

Placement of self-expanding, covered metal stents is the first choice of management in the palliative therapy of unresectable esophageal cancer. These stents have a much higher rate of stent migration (27\% compared with $8.7 \%$ of uncovered stents), and migration occurs more often at the gastroesophageal junction as the distal part of the stent is not fixed to the esophageal wall and projects freely into the lumen [1].
A partially covered esophageal stent with a S-type antireflux valve was placed in a 47-year-old man with advanced gastroesophageal adenocarcinoma, followed by relief of symptoms ( $\bullet$ Fig. $\mathbf{1}$ ). He vomited a fractured segment of the stent while on chemotherapy (to which the tumor responded) and then remained stable and asymptomatic. A contrast study revealed that the retained distal part of the stent had migrated proximally into the mid-

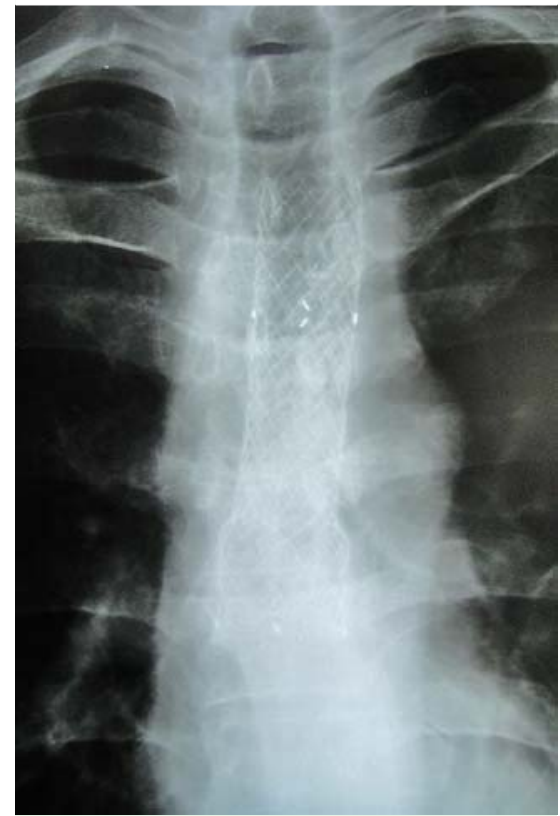

Fig. 2 Anteroposterior (AP) chest radiograph showing the retained, migrated fractured distal segment of the stent in the mid-esophagus.

esophagus. There was no contrast leak, and the contrast medium passed freely across the stent and distally, revealing tumor at the gastroesophageal junction ( $\bullet$ Fig. 2). On endoscopy a loose stent was found in the mid-esophagus and was carefully removed $(\bullet$ Fig. 3 ).

Stents mostly migrate distally due to peristalsis, although there are a few reports of proximal migration $[2,3]$. In the present case, proximal displacement of the stent may have occurred due to the force of vomiting against the antireflux valve of the covered stent at the gastroesophageal junction following shrinkage of the tumor with chemotherapy. The embedded proximal flange of the uncovered portion may have contributed to the fracture of the displaced stent. Other reports of fracture have implicated faulty material and thermal overstrain at laser treatment [4]. Removal of migrated stents is not always successful and may even be dangerous. Removal of distally migrated stents is thought to be unnecessary as these stents may be retained without risk of harm or they may pass spontaneously, with a low chance of impaction or perforation [5]. Proximal migration may be a case for re- 
moval of the stent as it may lodge in the nasopharynx or become a hazard for the airway [2,3]. Also, technically it may be easier to remove a proximally migrated stent than a stent that has migrated distally into stomach or beyond.

Endoscopy_UCTN_Code_CPL_1AH_2AD

Competing interests: None

\section{R. M. Gomes, S. Nagral, P. Agarwal}

Department of Surgical Gastroenterology, Jaslok Hospital and Research Centre,

Mumbai, India

\section{References}

1 Homann N, Noftz MR, Klingenberg-Noftz RD et al. Delayed complications after placement of self-expanding stents in malignant esophageal obstruction: treatment strategies and survival rate. Dig Dis Sci 2008; 53 (Suppl. 02): 334-340

2 Conti M, Ferraro P, Martin J et al. Esophageal stent migration into the trachea. J Thorac Cardiovasc Surg 2007; 134 (Suppl. 04): 1084-1085

3 Al-Zahid S, Clarke J, Roberts C. Chronic sinusitis and bilateral deafness as a presentation of oesophageal stent failure: case report. J Laryngol Otol 2009; 123 (Suppl. 04): 471 474

4 Doğan UB, Eğilmez E. Broken stent in oesophageal malignancy: a rare complication. Acta Gastroenterol Belg 2005; 68 (Suppl. 02): 264-266

5 Di Fiore F, Lecleire S, Antonietti M et al. Spontaneous passage of a dislocated esophageal metal stent: report of two cases. Endoscopy 2003; 35 (Suppl. 03): 223-225

\section{Bibliography}

Dol http://dx.doi.org/ 10.1055/s-0032-1308927

Endoscopy 2012; 44: E234-E235

(c) Georg Thieme Verlag KG

Stuttgart · New York

ISSN 0013-726X

\section{Corresponding author}

\section{R. M. Gomes}

Department of Surgical Gastroenterology Jaslok Hospital and Research Centre Mumbai

India

dr.gomes@rediffmail.com 